\title{
PROYECTO CURRICULAR \\ DE ESPECIALIZACIÓN \\ EN EDUCACIÓN MATEMÁTICA
}

\author{
Departamento de Matemáticas \\ Universidad Pedagógica Nacional
}

\begin{abstract}
What follows is a description of the Specialization program in Mathematics Education; a postgraduate program directed forward students with a Teacher Degree in Mathematics or Mathematics and Physics, or a Bachelor Degree in Mathematics. The objective of the program is the qualification of the Mathematics teacher's daily practice. The curriculum is developed during three semesters, each of which centers its attention on the didactics of a particular area of school mathematics. Students can choose three of any of the following areas:

Calculus, Algebra, Geometry, Probabiity and Statistics, and the Use of Technology in the teaching of school mathematics.
\end{abstract}

\section{INTRODUCCIÓN}

El proyecto curricular de Especialización en Educación Matemática, resultado de un proceso de recontextualización y renovación del programa actual de Especialización en Educación Matemática, será ofrecido por el Departamento de Matemáticas de la Facultad de Ciencia y Tecnología, la cual está conformada por los Departamentos de Física, Química, Biología, Matemáticas y Tecnología.

El Departamento de Matemáticas ofrece en la actualidad, además del programa de Especialización, otros programas académicos, cuales son, la Licenciatura en Matemáticas y la Maestría en Docencia de la Matemática. Estos programas, formulados, en su momento, a partir del reconocimiento de aspectos básicos de la problemática de la enseñanza de la matemática en los niveles básico y medio de nuestro país, se articulan y nutren entre si, retroalimentándose recurrentemente.

La actividad investigativa en el Departamento se ha centrado en los campos del diseño, análisis y desarrollo curricular y de la investigación didáctica, particularmente. Los docentes investigadores en el Departamento de Matemáticas no están adscritos a ninguno de los programas académicos que éste ofrece; los resultados y logros de la investigación que desarrollan alimentan a todos y a cada uno de los proyectos curriculares que ofrece y a las actividades de extensión que realiza.

La socialización y extensión a la comunidad se ha consolidado, de manera particular, con la participación permanente de los profesores y alumnos del Departamento en el Coloquio Distrital de Matemáticas, evento anual organizado, desde hace 16 años, en colaboración con la Universidad Nacional y la Universidad Distrital, en el Encuentro Anual de Geometría y sus Aplicaciones que organiza el Departamento de Matemáticas, desde hace 10 años, y recientemente, en las Conferencias Latinoamericanas en Educación 
Matemática, así como también a través del desarrollo de prácticas pedagógicas de los estudiantes en diferentes ambientes escolares, y en el ofrecimiento de cursos y talleres para profesores de la educación básica y media.

El trabajo investigativo y de extensión ha enriquecido la ubicación contextual del Departamento y de cada uno de los programas académicos que ofrece. La socialización de estas actividades y de sus resultados se da, además, a través de publicaciones como los cuadernillos del Coloquio Distrital de Matemáticas, las memorias del Encuentro de Geometría y sus Aplicaciones, la revista de la Facultad de Ciencia y Tecnología de la U.P.N., publicaciones de docentes del Departamento en revistas nacionales e internacionales, y a través de los demás medios de difusión de las actividades de la Universidad.

Dado el estrecho vínculo entre docencia, investigación y extensión que se ha establecido en las actividades que desarrolla el Departamento de Matemáticas, los diferentes espacios académicos y el desarrollo de la actividad investigativa propiamente dicha se constituyen en sí mismos en espacios de cualificación para sus docentes. Los profesores hacen igualmente uso de las oportunidades de cualificación que brinda la Universidad tales como la participación en cursos, talleres, encuentros, coloquios, seminarios nacionales e internacionales, etc. Esta forma de asumir la cualificación de los profesores se basa en la convicción de que la formación permanente de los docentes está estrechamente relacionada con la calidad de la actividad que éstos realizan y con su participación activa en la comunidad académica de docentes.

\section{ANTECEDENTES Y JUSTIFICACIÓN}

Dentro de los cuestionamientos que se han hecho a la enseñanza tradicional de las matemáticas en la escuela, se destacan:

- El modelo transmisionista que ignora el papel protagónico del estudiante.

- La concepción del profesor y el texto como únicas fuentes de verdad y de conocimiento.

- La percepción del saber matemático como la habilidad para desarrollar procesos y memorizar fórmulas.

- La idea generalizada de cómo el hacer matemáticas consiste en la ejecución mecánica de algoritmos.

- La visión de la matemática como un cuerpo de conceptos terminado y procesos aislados de otras ramas del saber.

Esta situación de la enseñanza tradicional fue el resultado de paradigmas investigativos psicológicos y pedagógicos, cuya vigencia ha caducado. Las investigaciones recientes en psicología cognitiva y Educación Matemática han generado nuevos paradigmas que a su vez han permitido avances notorios en el campo de la Educación Matemática con nuevos planteamientos sobre el quehacer profesional del docente, el análisis de la importancia de la comunicación y la forma de acceder a los conceptos matemáticos, entre otros aspectos. Particularmente, hoy se considera: 
- Los estudiantes y el profesor conforman una comunidad matemática en la cual todos participan activamente en la construcción significativa del saber matemático.

- La memorización ha de dar paso a procesos de razonamiento.

- El trabajo matemático se concibe como una actividad en la cual se conjetura, se crean y resuelven problemas, y se establecen conexiones entre las ideas y las aplicaciones de la matemática.

- La matemática se ha de ver, no sólo como una actividad cognitiva dentro de la matemática y para la matemática, sino como un instrumento que ayuda a resolver problemas sociales de la vida cotidiana.

- El maestro ha de ser un agente de cambio dentro de la comunidad en la cual está inmerso porque está activamente involucrado en la solución de los problemas que ésta posee.

El aprendizaje de las matemáticas implica la construcción de un conjunto de herramientas intelectuales indispensables para dar sentido a diversas situaciones de la vida cotidiana, las ciencias y las matemáticas. Algunas de estas herramientas son:

la habilidad para explorar, conjeturar y razonar lógicamente; la capacidad para resolver problemas no rutinarios; la competencia para comunicar ideas acerca de y por medio de las matemáticas; la destreza para conectar conceptos dentro de las matemáticas y entre las matemáticas y otras actividades intelectuales.

Los planteamientos de las nuevas concepciones de enseñanza y aprendizaje de la matemática, del conocimiento profesional especifico del profesor de matemáticas y los cambios que la escuela colombiana requiere, exigen el continuo perfeccionamiento del maestro en ejercicio y una educación continuada, concebida como la formación secuencial, en diferentes niveles; particularmente exigen perfeccionar docentes, de la educación básica y media, más hábiles en seleccionar tareas que capten el interés e intelecto de sus estudiantes, que ofrezcan oportunidades para profundizar la comprensión de la matemática estudiada y de sus aplicaciones, que dirijan sus clases de tal forma que promuevan en ellas la investigación y el desarrollo de ideas matemáticas, y que estén capacitado para usar los últimos adelantos tecnológicos y para impulsar a sus alumnos al uso de éstos, como herramienta en su trabajo.

Respecto a la Didáctica, en el programa de Especialización, aun cuando no se buscaba un desarrollo teórico, se trabajaban dos modelos didácticos. En los seminarios de profundización matemática se utilizaba un modelo de "auto reflexión", cuyo objetivo era cuestionar el conocimiento específico acerca de un tema e inducir, a partir de actividades que ponían en conflicto los conocimientos previos y despertaban el asombro de los participantes, la construcción matemática y el logro del aprendizaje significativo.

En los seminarios de Didáctica se presentaba otro modelo, basado en la propuesta de Higginson (1980), en el cual se visualiza la didáctica de la matemática en términos de las interacciones entre las disciplinas desde las cuales se asumen preguntas básicas como qué enseñar" "por que", "a quién y donde" y "cuándo y cómo". El "qué" hace referencia al contenido matemático u objeto de estudio, el cual se debe seleccionar con base en un amplio conocimiento del panorama de la disciplina, de los intereses de los estudiantes, de los requerimientos del plan de estudios y del contexto social, entre otros. El "cómo" reflexiona sobre el método para abordar el "qué" con el fin de lograr un mejor 
aprendizaje. El cómo" se considera determinado por el "qué" y es el objeto de estudio de la didáctica de la matemática. Particularmente el proyecto asumía la propuesta del Ministerio de Educación, producto de la renovación curricular de los años 80 , sin cuestionarla.

En la actualidad se reconoce que el conocimiento matemático, al igual que todo conocimiento es fruto de las experiencias de personas que interactúan en entornos particulares, en culturas diferentes y períodos históricos diversos. Este reconocimiento redimensiona el papel del docente de matemáticas, lo compromete con a función social de la escuela y lo induce a aprovechar su disciplina como herramienta intelectual esencial para dar respuesta a un sin número de opciones e intereses sociales que entrecruzan el mundo actual.

En la actualidad, la Didáctica de Matemática se considera una disciplina teóricopráctica de enfoque sistémico, con objeto propio de estudio y metodologías particulares de investigación. Fruto de sus desarrollos es el tratamiento didáctico particular para cada una de las áreas de la matemática. Desde esta perspectiva, se asume la necesidad de la renovación del proyecto curricular de la Especialización.

Como el Departamento de Matemáticas de la UPN es consciente que los programas de formación inicial ofrecidos hasta el momento, no han enfatizado en esta nueva orientación, compete a un proyecto curricular de especialización, proporcionar los espacios de cualificación y perfeccionamiento a este respecto. Las demandas sociales exigen cambios en la matemática escolar, que conlleven al desarrollo de valores democráticos, de mentes capaces de enfrentar los desarrollos tecnológicos y de comunicación que la sociedad exige.

\section{EL PROYECTO CURRICULAR DE ESPECIALIZACIÓN EN EDUCACIÓN MATEMÁTICA COMO PROYECTO DE INVESTIGACIÓN}

La necesidad de mejoramiento de la situación actual de la educación matemática en los niveles básico y medio en nuestro país, ha comprometido la reflexión profunda de profesores y estudiantes del Departamento de Matemáticas, sobre la complejidad de los aspectos que se deben conjugar en un proyecto curricular que pretenda incidir en la cualificación de la práctica profesional del docente de matemáticas en esos niveles.

Con base en el análisis sobre la forma de relación entre los procesos del conocimiento disciplinar específico y los procesos que se desarrollan en la práctica profesional misma, entendida ésta en todas sus dimensiones, se ha planteado el siguiente interrogante:

¿Logra el Proyecto Curricular de Especialización en Educación Matemáticas de la UPN, que atiende a la formación previa del licenciado en matemáticas y está estructurado con base en resultados de investigaciones en didácticas de áreas especificas de las matemáticas, modificar el conocimiento profesional para el desempeño de la labor docente en el aula?

Los profesores del Departamento de Matemáticas de la Universidad Pedagógica Nacional han considerado, para responder este interrogante, integrar al proyecto el estudio sobre: 
- El desarrollo histórico - epistemológico del saber matemático.

- Los elementos para la construcción de una didáctica en áreas particulares de las matemáticas.

- Las dificultades cognitivas asociadas al aprendizaje de áreas particulares de las matemáticas: concepciones, obstáculos, errores.

Como eje curricular del proyecto se propone el estudio de la didáctica de la matemática (de las diferentes áreas) y de sus desarrollos, conforme a los avances que el campo de la educación matemática ha logrado. Desde esta perspectiva, se impulsará en el proyecto los procesos de perfeccionamiento de los licenciados, integrados a las actividades propias de la investigación en el campo profesional del profesor, quien necesita conocimientos cada vez más especializados y fundamentados en el equilibrio entre una sólida formación en matemáticas con una buena formación profesional en didáctica de la matemática.

La hipótesis que se plantea es la siguiente:

Es posible perfeccionar y cualificar el desempeño profesional del licenciado en matemáticas, a través de su participación en un programa de especialización cuyo contenido y estructura se definen específicamente en el nuevo "Proyecto Curricular de Especialización en Educación Matemática del Departamento de Matemáticas de la Universidad Pedagógica Nacional".

El proyecto de investigación curricular se inscribe en la línea de investigación Investigación Acción Diagnóstica- Investigación Acción Empírica". La primera se enfoca hacia la recolección de datos, su interpretación, diagnóstico y recomendaciones para la acción, en tanto que la segunda se orienta hacia el diseño de acciones para el cambio. Las etapas fundamentales de la investigación del proyecto serán características de la Investigación Acción (planificación, acción, observación y reflexión).

La propuesta de evaluación del proyecto curricular, como proyecto de investigación, caracteriza a la evaluación como una actividad de autorregulación de procesos, fundamentada en el concepto de autoevaluación.

Específicamente, para la autoevaluación del proyecto curricular se han previsto las siguientes etapas:

- Planificación y diseño del programa: En esta fase se establecen los criterios para la autoevaluación.

- Organización de la autoevaluación: Se realiza en términos de la conformación de grupos de trabajo con responsabilidades específicas para conducir la autoevaluación de diferentes aspectos y la recolección y procesamiento de información específica requerida para la autorregulación.

- Conducción de autoevaluaciones: A lo largo del desarrollo del proceso se detectarán los problemas, las debilidades y las fortalezas del programa, lo cual permitirá tomar las decisiones e iniciar las acciones pertinentes para una autorregulación constante y oportuna. 
- Elaboración de informes: Estos informes deben dar cuenta de los procesos seguidos, de los análisis evaluativos y de las conclusiones en las que se incorporen los cambios, adecuaciones e innovaciones necesarios para continuar aproximándose al logro de los criterios de calidad esperados.

De otra parte, la autoevaluación del proyecto curricular comprende los siguientes aspectos:

- La pertinencia reflejada en ja declaración de propósitos del mismo, su razón de ser y los objetivos de aprendizaje que direccionan y le dan identidad al proyecto curricular.

- Los procesos: La autoevaluación permitirá analizar la organización del plan de estudios, su consistencia, equilibrio y pertinencia, así como juzgar los compromisos de trabajo y las acciones docentes, la investigación y la extensión, en relación con su coherencia con los propósitos del programa.

- Los resultados: Este aspecto de la autoevaluación está estrechamente relacionado con lo que se podría denominar el impacto del proyecto, medido a través de la modificación del conocimiento profesional para el desempeño de la labor docente en el aula.

En consistencia con la decisión de formular y asumir el proyecto curricular de Especialización como proyecto de investigación, los parámetros de su evaluación cumplirán los establecidos para un proyecto de investigación. En este sentido los resultados particulares que se esperan de su desarrollo son de una parte, la producción de conocimiento y de otra el impacto en los estudiantes usuarios del proyecto.

La producción de conocimiento se concretiza en los informes que cada uno de los equipos docentes encargados de los diferentes núcleos presenta. En razón a que cada uno de los núcleos del programa se alimentara de los resultados del trabajo investigativo que desarrollan los diferentes equipos de docentes del Departamento, colateralmente los informes de avances de estas investigaciones se consideraran productos del programa en tanto que se realizan para alimentarlo.

El impacto en los estudiantes se concretiza en el logro de las competencias formuladas.

\section{SUPUESTOS CONCEPTUALES}

Uno de los presupuestos que mejor permite planificar, profesionalizar y perfeccionar la función docente, uniendo teoría y práctica, es la concepción del diseño y desarrollo curricular como proyecto de investigación, considerando al mismo como objeto de discusión y debate.

Es bien aceptada la premisa de que el perfeccionamiento del licenciado y la caracterización del perfil del profesor especialista, están determinados por un diagnóstico preciso de la situación de la educación matemática en nuestro medio por un conocimiento cabal de la formación previa que tiene el licenciado y por las relaciones coherentes entre los ambientes en los cuales se forma y se perfecciona el profesor y la concepción de la práctica profesional misma. 


\section{Acerca de la didáctica de las matemáticas}

Como ya se anotó, la concepción que sobre la didáctica manejó el programa de especialización admite una reformulación de éste que requiere la incorporación de la teoría didáctica correspondiente a cada área de la matemática La didáctica de la matemática se fundamenta en el conocimiento profundo de la disciplina y en el conocimiento del sistema didáctico. Su acción ha de centrarse en el análisis y la búsqueda de respuestas a la problemática de la enseñabilidad de la matemática escolar y de la educabilidad del discente.

En la reflexión teórica acerca de la didáctica el "qué matemático" se convierte en objeto de análisis puesto que los conocimientos matemáticos no sólo hacen referencia a contenidos específicos de su estructura actual, sino también a la actividad mediante la cual se construyen y a la forma como han evolucionado. De este modo los conceptos matemáticos se perciben dinámicos y no como un conjunto fijo de conocimientos. Quien "hace matemáticas", comete errores, conjetura, abstrae, generaliza y comprueba para luego comunicar los resultados a la comunidad de especialistas, bajo esquemas acordados, escondiendo el proceso usado en su generación, proceso valioso en la formación del aprendiz de matemática. Inherentes al conocimiento matemático van insertos no solamente los conceptos sino también la representación simbólica y los procesos de desarrollo y validación. Aprender matemática no consiste en acumular información para resolver ejercicios. Es un proceso en el cual debe vincularse el individuo, mediante vivencias significativas, en el desarrollo de los conceptos, es decir, "hacer matemática".

El sistema didáctico hace referencia a las interrelaciones entre los profesores, los estudiantes y el conocimiento matemático, teniendo en cuenta, además, el mundo exterior a la escuela conformado por la sociedad, los padres y los matemáticos entre otros. EI papel del profesor deja de ser el de autoridad para transformarse en guía que recontextualiza el saber que quiere comunicar a sus alumnos con el propósito de propiciar en el aula de clase una actividad verdaderamente científica. El estudiante asume un papel activo en su aprendizaje para construir conocimiento, formularlo, validarlo, incorporarlo a su estructura cognitiva, y usarlo en futuras situaciones.

La investigación didáctica busca la sistematización de teorías y nuevas herramientas para acceder a los conceptos matemáticos. Aporta elementos teóricos para el perfeccionamiento de la actividad educativa, teniendo en cuenta los criterios de enseñabilidad de la disciplina y de la educabilidad del estudiante.

\section{Acerca de lo pedagógico}

La decisión de asumir un enfoque pedagógico que oriente y determine el Proyecto Curricular, debe tener en cuenta la confrontación teórica y práctica que desde tiempo atrás contrapone dos perspectivas pedagógicas. En una perspectiva se quiere instruir y formar al estudiante desde el exterior, por lo que el acto pedagógico se concibe como una actividad de dirigir, modelar y equipar de conocimientos al estudiante. La antítesis de esta perspectiva es la de considerar que el estudiante lleva en sí mismo los medios para lograr su propio desarrollo y que toda acción que intervenga, desde el exterior, no hará sino deformarlo y obstaculizarla. Estas dos perspectivas, fortalecidas por desarrollos teóricos, han conducido a polarizar el debate sobre lo pedagógico como un campo de posicionamiento exclusivo y a veces dogmático. 
El equipo de profesores del Departamento de Matemáticas es consciente de la confrontación que suscitan estas posiciones y acorde con los criterios descritos, considera que toda situación pedagógica es cambiante, compleja, responde a fines y metas, y presenta conflictos. Por consiguiente, no existe, ni puede existir, una única y reconocida teoría pedagógica científica desde la cual se orienten unívocamente los medios y las reglas para alcanzar los fines propuestos.

La premisa anterior, aunada a la convicción que son "lo pedagógico" y "lo didáctico" el objeto de estudio y de trabajo del profesional de la educación, nos lleva a establecer que asumimos la tarea de construir un enfoque pedagógico como una tarea reflexiva y compleja, que se desarrolla en escenarios singulares, claramente determinados por los contextos cargados de conflictos de valor, que requieren opciones éticas y políticas diversas (Pérez, 1993). Consideramos que lo pedagógico trasciende ampliamente el carácter exclusivo de la enseñanza, para integrar la reflexión sobre la complejidad de las relaciones que determinan el desempeño del profesor. El perfeccionamiento del profesor no está enmarcado exclusivamente desde la perspectiva de la adquisición puntual de conocimiento, para responder a exigencias de los diferentes ambientes de formación. En términos generales, podemos afirmar que la profundización de conocimientos por parte del estudiante-profesor, es un proceso que adquiere su verdadero valor cuando los conocimientos se integran al desarrollo de la propia personalidad y cuando se convierten en competencias significativas para su desempeño profesional.

Los supuestos de la intervención pedagógica, en esta propuesta. se caracterizan por la incorporación de conocimiento significativo y relevante para comprender y experimentar, en cada contexto singular (ambiente de formación), procesos de reflexión en la acción, para desarrollar competencias en el estudiante que le permitan, en sus actuaciones de la vida profesional, elaborar diagnósticos, valorar sus componentes, diseñar estrategias alternativas y prever, en lo posible, el curso de futuros acontecimientos. De esta manera, el conocimiento académico, teórico y científico se integra significativamente en esquemas de pensamiento más genéricos, en contraposición a la concepción de adquisición de conocimiento en parcelas aisladas.

Además de la postura expuesta anteriormente, que caracteriza el enfoque pedagógico que asume el Departamento de Matemáticas de la UPN, a continuación se presentan las concepciones que ha elaborado sobre otros núcleos del saber pedagógico y el análisis de las interrelaciones entre éstos, análisis que enriquece la reflexión pedagógica en que se empeña:

\section{Acerca de la enseñabilidad}

Las condiciones que determinan las posibilidades de la enseñanza del conocimiento matemático están dadas por las funciones sociales que se le asignen al proyecto de enseñanza y por la concepción sobre la naturaleza del conocimiento matemático. Un ejemplo de esta relación, se encuentra en la propuesta de formación de profesores de la década del setenta, cuyo modelo de enseñanza se concibió bajo el carácter exclusivo de formación de matemáticos y en el cual los objetos matemáticos se consideraban independientes de los sujetos, con una naturaleza absoluta, incuestionables. La matemática era considerada como cúmulo de verdades absolutas, eternas e inmodificables.

La propuesta actual, por el contrario, si asume el carácter de proyecto social de la enseñanza de las matemáticas en el programa de perfeccionamiento de profesores y 
espera que éste cumpla además otros varios fines. Además del conocimiento y comprensión del cuerpo de teorías matemáticas y de su lenguaje científico, debe aunarse la búsqueda de la interpretación de las matemáticas mismas. El profesor de matemáticas debe elaborar transformaciones de los conocimientos matemáticos para recontextualizarlos, re-personalizarlos, re-temporalizarlos en los contextos de su actuación como profesional de la docencia de la matemática.

De esta manera, la enseñabilidad de las matemáticas es una elaboración del profesor con base en sus compromisos epistemológicos, pedagógicos y didácticos y, que depende además de sus estructuras conceptuales, estéticas, actitudinales y axiológicas. Los profesores de matemáticas enseñan esta disciplina de acuerdo con ciertas ideas que ellos tienen acerca de las matemáticas y de cómo éstas deben ser aprendidas parlas estudiantes; en la práctica de la enseñanza de las matemáticas, el profesor continuamente toma decisiones respecto al contenido y la forma de presentación en el salón de clases.

Una de las funciones del profesor de matemáticas es precisamente la de construir matemáticas para la escuela, y esto de suyo determina la enseñabilidad de las mismas.

\section{Acerca de la educabilidad}

Siguiendo con el desarrollo de los criterios planteados en este documento, es claro entonces que asumimos que los alumnos aprenden y asimilan teorías, actitudes y desarrollan competencias, no sólo como consecuencia de la socialización e intercambio de ideas y conocimientos explícitos en el currículum, sino también, por las posibilidades de interacción social que se propicien en la institución en general y en el aula en particular y por la estructura de la tareas que se propongan.

De esta manera, concebimos el aprendizaje, en función de la comunicación y mediado por el aprendiz y las circunstancias reales en que se desarrolla la intervención educativa.

Quien aprende reconstruye y construye nuevos significados, formas de significar y de actuar intencionalmente; de esta manera, se halla en posicionamientos diferentes desde los cuales atribuir otros sentidos al mundo. En este sentido, toda aproximación al saber es realizada desde las estructuras conceptuales, metodológicas, estéticas, actitudinales y axiológicas que cada quien ha elaborado, y es desde ellas que se hacen las respectivas lecturas, atribuyéndoles el sentido que se considera apropiado y pertinente. Puesto que por muy equivalentes que sean esas estructuras entre los miembros de la comunidad, no son estrictamente iguales unas a otras, es de esperarse que las lecturas no coincidan y, por tanto, que existan siempre controversias y la necesidad de negociar acuerdos programáticos. (Gallego y Pérez, 1999).

La interacción y la cooperación movilizan la reflexión sobre la acción, la cual es considerada como un mecanismo básico para acceder a los niveles superiores de abstracción e internalización. Es esta la razón por la cual, en la educabilídad, está implícito el reconocimiento del otro y que el acto de educar, que se desprende de esta concepción, se traduce en un acompañamiento del educando para que ingrese de si o por sí mismo en el orden que se le ofrece. Esta concepción no desconoce las lógicas de las intencionalidades curriculares en la dirección incuestionable de que todo educando ha de, finalmente, incorporarse críticamente al proyecto cultural, social, político y económico con miras a realizar su proyecto ético de vida. 


\section{Acerca de la interdisciplinariedad}

La interdisciplinariedad tanto de la matemática como de la educación matemática están claramente definidas. La historia y la epistemología de las matemáticas cobran todo su valor en el desempeño del profesor de matemáticas, por cuanto es en la comprensión de la construcción de diferentes saberes y conceptos matemáticos, en el estudio de los obstáculos y errores que se sortearon en el proceso de gestación de dichos conceptos, y la comprensión de las condiciones históricas socioculturales que rodearon su desarrollo, donde el profesor de matemáticas adquiere el verdadero sentido y comprensión, tanto de la actividad matemática misma, como de las variantes que intervienen en la construcción comprensiva de ella.

Las disciplinas anteriormente mencionadas amplían la perspectiva sobre la naturaleza de las matemáticas. La construcción inductiva junto con la resolución de problemas como fuentes de la generación del conocimiento matemático se integran a la aproximación deductiva como herramientas esenciales tanto en el ambiente correspondiente a la formación disciplinar del profesor de matemáticas como en la tarea de diseñar y desarrollar matemáticas escolares.

No se puede dejar de mencionar el aporte de la psicología, la antropología, las ciencias naturales, la biología y en particular la neurología, la filosofía, la historia de la matemática, la lógica, la lingüística y la informática a la comprensión sobre la naturaleza de las matemáticas y la relación conocimiento-profesor-alumno.

Como se puede deducir, las preguntas y los referentes teóricos que soportan el Proyecto, nos remiten a campos de conocimiento muy diversos y a marcos teóricos muy variados y complementarios. Puesto que los problemas que plantean la formación y el perfeccionamiento de profesores de matemáticas no pueden ni desmenuzarse, ni ser resueltos por una sola área de conocimiento, el campo de formación no puede ser considerado como una simple suma o yuxtaposición de áreas de conocimiento. La búsqueda de la interdisciplinariedad se constituye en un reto para el mismo proyecto.

\section{PROPÓSITOS DEL NUEVO PROYECTO CURRICULAR}

El proyecto curricular de Especialización en Educación Matemática, inscrito en el marco de la nueva normatividad educativa, busca responder, en lo posible, a las necesidades propias de la enseñanza de las matemáticas en nuestro contexto, particularmente en los niveles de la educación básica y media.

En concordancia con las finalidades que plantea la Ley General de Educación, especialmente en lo referente a la formación de educadores de la más alta calidad científica y ética, al desarrollo de la teoría y la práctica pedagógica como parte fundamental del saber del educador y al fortalecimiento de la investigación en el campo pedagógico y en el saber específico y, consistentemente con la misión y propósitos de la UPN presentados en su proyecto educativo institucional y con los objetivos particulares del Departamento de matemáticas, se plantean como propósitos específicos del proyecto curricular de Especialización en Educación Matemática:

- Ofrecer un programa académico de especialización, como una opción de perfeccionamiento para el licenciado, a través del cual pueda modificar su conocimiento profesional. 
- Sistematizar el perfeccionamiento y la capacitación de los docentes de matemáticas y estructurar esta última alrededor de metas claramente definidas.

Particularmente, se plantean como propósitos formativos del proyecto curricular de Especialización los siguientes:

- Propiciar la reflexión sobre la problemática contemporánea de la enseñanza de la matemática y favorecer la toma de conciencia sobre las principales dificultades que afectan el aprendizaje de esta disciplina, con miras a generar en el profesor, y consecuentemente en sus estudiantes, una nueva actitud frente a la matemática y a su aprendizaje.

- Generar estrategias para la profundización en el estudio de la didáctica de las matemáticas, a partir de la reflexión y sistematización de sus prácticas cotidianas.

- Formular proyectos de aula que transformen la docencia convencional y que, a través de la investigación didáctica, permitan aproximarse a la solución de los problemas educativos derivados de la actividad docente específica.

- Contribuir a la conformación de grupos de trabajo de profesores de matemática de los niveles básico y medio que puedan incidir favorablemente en el mejoramiento de la práctica profesional del docente de esta disciplina, en nuestras escuelas y colegios.

\section{ESTRUCTURA CURRICULAR}

En razón a que el proyecto de Especialización en Educación Matemática está encaminado a generar procesos de formación y de perfeccionamiento en la acción, a que el enfoque desde el cual se plantea la cualificación de los participantes parte del conocimiento de la formación inicial del licenciado y a que apunta a la generación de condiciones adecuadas para un mejoramiento de la enseñanza de las matemáticas, en particular, en las instituciones en donde están vinculados los estudiantes del programa, el plan de estudios es flexible y receptivo a las condiciones y preferencias de tales instituciones y a las de los profesores participantes.

Como ya se anotó anteriormente, el proceso actual de renovación de los proyectos de licenciatura, especialización y maestría del Departamento de Matemáticas se enfocó, entre otras, desde la perspectiva de ofrecer al estudiante que 0pta por la profesión de educador, la posibilidad de formarse inicialmente (proyecto curricular de licenciatura), perfeccionar y cualificar su práctica profesional profundizando en el conocimiento profesional propio del profesor de matemáticas (proyecto curricular de Especialización en Educación Matemática) y encaminarse hacia la investigación (proyecto curricular de Maestría en Docencia de la Matemática).

Es, desde esta perspectiva de formación y perfeccionamiento continuo que se define la estructura del proyecto curricular de la Especialización alrededor de la didáctica de la matemática como eje central y que se definen, en coherencia con los lineamientos curriculares del Proyecto Político Pedagógico Institucional de la UPN, los núcleos temáticos e integradores y los ambientes vivenciales de formación que imprimirán en el estudiante las características particulares de unnal de la educación formado en la UPN. 
El núcleo integrador de la estructura curricular del proyecto enfoca el análisis y la profundización de los procesos de formalización, construcción, explicación, sistematización y contextualización, caracterizados por el conocimiento didáctico

El diseño curricular del proyecto se expresa en la caracterización de los ambientes de formación disciplinar específica, pedagógico, científico e investigativo, deontológico y en valores humanos y lingüístico en los cuales se desarrolla y, en el plan de estudios que caracteriza los ejes curriculares, los núcleos integradores y temáticos del mismo, así como en la concepción de evaluación que acoge.

\section{Ambientes de formación}

Con respecto a estos ambientes, un estudiante del Programa de Especialización del Departamento de Matemáticas, vivenciará particularmente los siguientes:

Formación disciplinar específica

El ambiente de formación disciplinar específica se centra en la Didáctica de las matemáticas. En particular se enfatiza en:

- Los procesos de formalización, globalización y síntesis del conocimiento matemático, los cuales en la licenciatura han sentado las bases para generar actividades investigativas propias del docente de matemáticas y cuyo estudio profundo y sistemático le permitirá al licenciado, que accede al proyecto, comprender y desarrollar didácticas específicas en cada área.

- El acercamiento del docente a desarrollos en la disciplina, con el objeto de estudiar la pertinencia y viabilidad de transformarlos en conocimientos escolares.

- La concepción de las matemáticas y de su enseñanza como actividades constructivas. Esta concepción conllevará al estudiante a vivenciar todas aquellas actividades propias, tanto del quehacer matemático como del quehacer del profesor de matemáticas

- Un análisis histórico-epistemológico de la evolución del conocimiento matemático

\section{Formación pedagógica}

El ambiente de formación pedagógica por cuanto todos los proyectos curriculares que ofrece el Departamento se enmarcan en un campo de acción cuya disciplina fundante es la pedagogía y orientan sus acciones a la formación y perfeccionamiento de profesionales de la educación capaces de promover acciones individuales y colectivas y de comprender y actuar ante la problemática educativa en la perspectiva del desarrollo integral y humano.

En el proyecto de la Especialización, el docente comparte con sus colegas condiscípulos la experiencia de «enseñar» matemáticas, profundiza en su formación teórica, actualiza sus conocimientos incorporando, a sus saberes, los desarrollos del campo de la educación matemática particularmente los referidos del conocimiento matemático y a la didáctica de las diferentes áreas de la matemática escolar. 


\section{Formación investigativa}

El ambiente investigativo, subyace en todos los demás, por cuanto en cada uno de ellos se prioriza la generación de actitudes como las de análisis, creación, síntesis, conjetura, sistematización, etc. Este ambiente, al igual que el de formación pedagógica, permea todos los espacios académicos del Departamento.

El proyecto curricular de Especialización reconoce el papel motivador preponderante que tiene el estudio del desarrollo histórico de la matemática y sus conexiones con las demás ciencias, desde la perspectiva de estudiar situaciones en las cuales se han generado y desarrollado conceptos matemáticos. Todos los espacios académicos del proyecto de Especialización se caracterizarán por su orientación hacia las problemáticas en el aula y contribuirán, de manera decisiva, en la formulación de los proyectos que presenten los estudiantes al finalizar cada periodo académico. Por cuanto el programa está alimentado por los desarrollos y avances investigativos de los equipos de profesores del Departamento de Matemáticas, el estudiante-profesor se verá enfrentado a los procesos propios de una investigación.

\section{Formación deontológica y en valores humanos}

El de formación deontológica y en valores humanos, caracterizado por la práctica vivencial cotidiana que se interioriza para construir la conciencia de cómo todo saber es intersubjetivo y obedece a creaciones colectivas que conllevan aportes, acuerdos, reconocimientos y compromisos, se constituye en la praxis que orienta y determina las prácticas sociales de construcción y negociación colectiva. El Departamento no desconoce la necesidad de referentes teóricos que contribuyan a la formación de valores, pero es consciente que sólo la vivencia de éstos, el ejercicio de la aceptación de la diferencia, el dialogo y búsqueda de la equidad y aceptaciones cotidianas y pedagógicas permite adquirir el significado y sentido de la formación ética y necesaria para el desempeño del ciudadano colombiano. El ambiente de formación deontológica es objeto permanente de trabajo en el Departamento, en tanto que la reflexión sobre cómo todos los saberes están inmersos en un contexto más amplio, el de la cultura, la cual es fundamento de la nacionalidad e involucra, además, valores éticos, estéticos, morales, intelectuales, democráticos y educativos entre otros, es cotidiana.

En el proyecto curricular de Especialización se contribuye particularmente al afianzamiento y crecimiento de la conciencia profesional del educador matemático.

\section{Formación lingüística}

El ambiente de formación lingüística enfatiza en el uso de competencias lingüísticas y comunicativas necesarias para el aprendizaje y para la enseñanza, para el fomento el debate y la argumentación, y se expresa, particularmente en el programa, en el estudio de informes de investigación, de artículos y comunicaciones, tanto en español como en inglés, y de temas relacionados con las didácticas específicas que se trabajan. La presentación y sustentación de ensayos y proyectos de aula, exige, por parte del estudiante, el dominio del lenguaje oral y escrito. 


\section{PLAN DE ESTUDIOS}

\section{Ejes curriculares}

El plan de estudios de la Especialización, en concordancia con los propósitos del proyecto curricular tiene como eje central la didáctica de la matemática y como ejes curriculares los siguientes:

- El desarrollo histórico-epistemológico del saber matemático.

Los elementos para la construcción de una didáctica en un área particular de las matemáticas.

- Las dificultades cognitivas asociadas al aprendizaje de un área particular de las matemáticas: concepciones, obstáculos, errores.

\section{Núcleos temáticos}

El plan de estudios está conformado por tres grandes núcleos temáticos que se desarrollan, cada uno, en un periodo académico de un trimestre, sin disgregación de asignaturas, con miras a lograr una alta intensidad en la problemática que se trabaja y un mínimo de diversidad en cuanto al estudio simultáneo de otros núcleos. Cada núcleo se pandera académicamente en 12 créditos.

El principio de flexibilidad en que se soporta el plan de estudios permitirá, de una parte, incrementar la proyección, la apertura, el rendimiento social, el impacto, la pertinencia y la cobertura del proyecto, y de otra parte le permitirá al estudiante, que conservando los ejes curriculares del proyecto, personalice el contenido del plan según sus propios intereses y necesidades, seleccionando cursar, parlo menos, tres de los siguientes núcleos:

- Didáctica del cálculo.

- Didáctica del álgebra.

- Didáctica de la geometría.

- Didáctica de la probabilidad y la estadística.

- Didáctica de la aritmética.

- Uso de la informática en la enseñanza de las matemáticas escolares.

Al cursar cada uno de los núcleos propuestos, se pretende que el estudiante:

- Profundice en el conocimiento de los elementos teóricos fundamentales para la comprensión y desarrollo de las didácticas particulares de cada área de la matemática.

- Identifique e interprete conceptualmente los elementos técnicos y metodológicos necesarios para configurar y estructurar el respectivo proyecto de aula.

Particularmente se espera de los egresados las siguientes competencias:

- La generación de propuestas de aula, consistentes e innovadoras que, atendiendo al contexto particular de su escuela, propendan por el logro de mejores niveles de aproximación al conocimiento matemático en niños y jóvenes. 
- El reconocimiento a la importancia y pertinencia de la gestación de grupos de trabajo en su escuela o zona (y de la participación activa en los mismos), que aborden el estudio de la didáctica de la matemática y propicien la sistematización y socialización de las prácticas docentes cotidianas.

- El desempeño integral y comprometido como docente de matemáticas.

Como ya se expresó, en cada uno de los núcleos temáticos del plan de estudios se trabajará la didáctica de un área específica de las matemáticas escolares. Los contenidos curriculares particulares de cada núcleo están determinados por los avances y desarrollos que alcancen, en cada área, los diferentes equipos que han conformado, según sus líneas de trabajo, los profesores del departamento.

El contenido que se prevé para cada uno de los núcleos es el siguiente:

\section{Núcleo: Didáctica de la Geometría}

El grupo de estudio del Departamento de Matemáticas, que centra su preocupación en la Didáctica de la Geometría, considera prioritario ofrecer a los profesionales de la Educación Matemática en ejercicio, un programa de perfeccionamiento en geometría en donde, además de cubrir los vacíos de formación que hayan podido tener en esta área durante la etapa de formación obligatoria, se presente una panorámica de los desarrollos investigativos en este campo y se de lugar a la reflexión sobre las posibles formas de recuperar los espacios de formación en geometría de sus educandos, espacios que las reformas curriculares de los años cincuenta y sesenta a nivel mundial y setenta en nuestro país, constriñeron al grado de casi suprimir la geometría de la escuela.

Hasta el momento el grupo de estudio viene trabajando en un modelo para la enseñanza de la geometría que seria el eje sobre el que se fundamentaría las actividades de formación que propondría el programa. El modelo está sustentado en la investigación sobre el razonamiento geométrico de Van hiele, recoge propuestas similares realizadas por las escuelas norteamericana e Israelí e incorpora el uso de la tecnología como herramienta de apoyo a la conceptualización geométrica.

\section{Núcleo: Didáctica del Cálculo}

Los conceptos de límite, derivada e integral son contenidos que se contemplan como necesarios en la enseñanza del Cálculo en la Educación Media. Su introducción se hace en este nivel como preparación para la matemática universitaria. Sin embargo en los primeros semestres se convierte en uno de los cursos con mayor grado de reprobación. Esta situación compartida por casi todos los sistemas de enseñanza, ha conducido a que su problemática sea considerada como uno de los problemas de investigación para la Educación matemática. La razón reside en que el Cálculo es una de las herramientas matemáticas más poderosas para modelar diversos fenómenos de la vida científica y cultural. A lo anterior se suma que es reconocido como un puente necesario hacia el pensamiento matemático avanzado.

Particularmente en a educación universitaria colombiana, el problema ha sido abordado desde la perspectiva de la investigación (Gómez, Carulla, y otros, 1990), y aunque en la educación media se encuentran algunas innovaciones en el aula es necesario enfocar el problema, a este nivel, desde la perspectiva de la investigación en educación matemática, como uno de los elementos esenciales para los procesos de diseños y desarrollos curriculares que en la actualidad adelanta la comunidad de 
profesores de matemáticas, puesto que determina las posibilidades reales de mejora de la calidad de la educación matemática en el país.

Un grupo de estudio del Departamento de Matemáticas que centra su preocupación en la didáctica del Cálculo, considera prioritario ofrecer a los profesores de matemáticas, un programa de perfeccionamiento fundamentado en la Didáctica del Cálculo, que aborde algunas de las siguientes temáticas, resultado de sus primeros acercamientos al estudio de la Didáctica del Cálculo.

El estudio, representación y solución de situaciones en las cuales están presentes nociones que sirven de fundamento a la construcción de los conceptos del Cálculo, como son:

— Variación y cambio: patrones

- Relación entre variaciones: La función como dependencia.

- Procesos de aproximación: el error

- Procesos infinitos.

—El continuo numérico: los números reales.

Problemática de la enseñanza y aprendizaje, en la escuela, de los conceptos del cálculo infinitesimal. Cálculo.

El pensamiento avanzado propio de la construcción de los conceptos básicos del

Estudio de investigaciones actualizadas sobre la enseñanza y el aprendizaje de los conceptos del Cálculo Infinitesimal.

\section{Núcleo: Didáctica del Álgebra}

Los conceptos de operación, función y estructura algebraica están presentes en la matemática de la educación básica y media. Sin embargo, muchos estudiantes, apenas manejan algoritmos en forma mecánica, sin dar sentido ni razones que justifiquen su acción sobre los elementos de un conjunto. Si esto es, de par, si, un indicativo inquietante en el aprendizaje, mayores son las deficiencias cuando de analizar, plantear y resolver problemas se trata. Es aquí donde aparecen las mayores dificultades para el estudiante. Parece que la falta de situaciones que le permitan hacer conjeturas de resultados generalizadas y su simbolización, la caracterización de estructuras algebraicas, la coherencia lógica de los procesos, le impiden enfrentar los retos que ofrece la solución de problemas.

La concepción de un álgebra sustentada en la pedagogía, con una didáctica específica, será uno de los propósitos de este núcleo en la Especialización, con el ánimo de superar las dificultades expuestas.

Tópicos centrales que estarían presentes en este núcleo:

Sistemas numéricos: estructuras algebraicas de los conjuntos de números enteros, racionales, reales y complejos.

Teoría de grupos. Construcción de grupos finitos y su aplicación para el desarrollo de procesos didácticos. Modelos geométricos para grupos finitos y 
La estructura de anillo y campo como fundamento teórico del álgebra elemental.

Teoría de ecuaciones.

\section{Núcleo: Didáctica de la Aritmética}

Tradicionalmente, la aritmética en la escuela se ha considerado como el ámbito en el cual se adquieren destrezas para la realización de cálculos numéricos y resolución de problemas, en el marco de las operaciones básicas de los conjuntos numéricas $\mathrm{N}, \mathrm{Z}$ y $\mathrm{Q}$. El énfasis puesto al estudio de los conjuntos numéricos ha ido cambiando a través de las diferentes propuestas curriculares, Una nueva perspectiva intenta desplazar la organización temática, que se centra en la teoría de conjuntos, y en la presentación formal de las estructuras numéricas, en la cual cada conjunto numérico surge como respuesta a problemas de tipo matemático. Ahora, se trata de aprovechar que la aritmética contribuye a ampliar la capacidad matemática, a través del desarrollo del sentido numérico y del razonamiento aritmético y proporcional, y que además aumenta las competencias comunicativas propias en este dominio.

Tópicos centrales que estarían presentes en este núcleo:

Tratamientos curriculares que posibiliten la comprensión de los números y las operaciones, así como la inclinación a usarlos de forma flexible para hacer estimaciones numéricas, desarrollar estrategias operativas útiles y resolver problemas.

Complejidad de la construcción conceptual del número. Perspectivas histórico-críticas en su evolución: del número natural al número entero, de la fracción al racional, etc.

Investigaciones relativas a la resolución de problemas aritméticos, clasificación de problemas, obstáculos y dificultades en su aprendizaje.

Aproximación al álgebra a través de la aritmética. La teoría de números y su potencial para desarrollar competencias de generalización, búsqueda de patrones de regularidad y formas inductivas de aproximación al conocimiento.

La calculadora como herramienta de desarrollo del pensamiento aritmético.

\section{Núcleo: Didáctica de la Probabilidad y Estadística}

En la actualidad la toma de decisiones sobre cualquier tópico se fundamenta en el análisis profundo de los datos pertinentes. En el mundo de hoy "La nacionalidad ya no puede seguir siendo identificada con la certeza, ni tampoco la probabilidad con la ignorancia" (Prigogine, 1986, pág. 183).

La probabilidad es característica propia de los razonamientos no demostrativos y determinar la validez y significado de dichos razonamientos es papel de la filosofía de la probabilidad. El currículo actual de la Educación Básica y Media considera como parte importante de la formación del futuro bachiller el conocimiento de las herramientas probabilísticas y estadísticas para la mejor comprensión de los fenómenos naturales y sociales. Se ha hecho énfasis en la parte — dejando de lado la parte inferencial, por ello se hace necesario contribuir a la formación de los docentes en fundamentos de estadística y probabilidad y en su aplicación inferencial. 
El objetivo del núcleo es facilitar la comprensión conceptual de la probabilidad y de la estadística y de las dificultades de su aprendizaje, así como de los métodos requeridos para su enseñanza.

El curso requiere trabajar con los docentes en tres niveles de reflexión:

La estructura matemática de la probabilidad y la estadística.

Conceptos, métodos y diagramas o medios de representación, es decir, el propio contenido eslocástico.

Los contextos de actividad y aprendizaje para los alumnos.

Medios especiales de representación, sistemas de tareas, y todos los instrumentos que permitan el aprendizaje de los alumnos por un camino significativo.

La perspectiva de la actividad del profesor sobre el proceso de enseñanza.

Planificación, organización, desarrollo modificación y evaluación de los procesos de aprendizaje y enseñanza del conocimiento eslocástica.

\section{Núcleo: Uso de la Informática para la enseñanza de las matemáticas escolares}

El computador es una herramienta poderosa que ayuda en el proceso de aprendizaje de la matemática. La facilidad de graficar y de realizar movimientos y simulaciones permite un buen acercamiento a muchos conceptos de la geometría, el álgebra y el cálculo escolares. Procesos lentos y rutinarios se mejoran con el empleo de programas que por su presentación y herramientas disponibles facilitan la labor del docente.

Una componente básica en el trabajo del especialista en educación matemática de hoy y del futuro es a utilización de los computadores en la enseñanza de la misma, por ello se hace necesario que el docente conozca las posibilidades que este tipo de paquetes ofrecen.

Es importante ver que la computación permite al estudiante representar en su propio ambiente de objetos cada nueva noción y concepto que aprende y a través de esta tarea y de la representación misma conseguir un mayor acercamiento a la matemática. Por ejemplo un lenguaje como LOGO, permite al niño y al adolescente explorar, representar y plantarse conjeturas que luego pueden ser despejadas con la orientación del docente.

El objetivo central de este núcleo es capacitar al docente para el empleo de los computadores en sus propias clases de matemáticas como herramienta que ayude en el proceso de aprendizaje. Para conseguirlo se requiere un conocimiento de la máquina y de su funcionamiento y el manejo profundo de uno o más paquetes de aplicación.

En el núcleo se trabajan dos elementos: uno de fundamentación en informática en la cual se desarrollen temas como la arquitectura básica de los microcomputadores los lenguajes de programación y las posibilidades y limitaciones de las máquinas y otro de aplicación de paquetes comerciales para la enseñanza de la matemática.

Dependiendo de los intereses de los estudiantes se pueden utilizar algunos de los siguientes paquetes: 
Logo (versión Terrapín de Harvard 1998). Con el cual se hacen construcciones geométricas, elementos de pre-geometría, introducción a los números enteros, elementos de trigonometría y de geometría analítica.

Derive: herramienta que permite el manejo de funciones y todas sus propiedades, gráficas y aproximaciones.

Matlab: herramienta que ayuda a la introducción de conceptos del álgebra lineal para el grado noveno, trabajo con funciones y gráficas, aplicaciones estadísticas, estudio de elementos de cálculo.

Mathematíca: paquete con el cual se consigue desarrollar conceptos alrededor de cualquier tema de las matemáticas escolares, permitiendo que el maestro elabore sus propios programas y guías para el trabajo con los alumnos.

Cabri, programa exclusivo par el aprendizaje de la geometría, que por la riqueza de opciones que ofrece permite trabajar con mucho éxito la geometría del bachillerato y proponer interesantes problemas que motivan y estimulan a los alumnos.

\section{METODOLOGÍA Y ESTRATEGIAS DIDÁCTICAS}

El proyecto curricular de Especialización parte del supuesto que el docente posee un saber. Los espacios formales están diseñados para cualificar sus experiencias y prácticas, además de abrir nuevos horizontes de significados que lo lleven a integrar en su quehacer los avances en la didáctica de la disciplina, particularmente buscan que el estudiante vivencie nuevas experiencias de aprendizaje, la experiencia de construir conocimiento y de "aprender a aprender".

El desarrollo del programa académico se realiza a través de actividades de tipo presencial, de estudio independiente y dirigido y, de tutorías.

Las actividades presenciales se desarrollan mediante exposiciones, conferencias, discusiones, debates, estudios de casos, talleres y simulaciones.

Las actividades de trabajo independiente dirigido comprenden lecturas especializadas con guías de estudio, que los estudiantes deben procesar en forma particular y que constituyen la base sobre la cual se realizan las actividades presenciales.

Las tutorías se ofrecen para orientar a los estudiantes en la planeación, diseño y desarrollo del trabajo que cada quien presentará al finalizar cada período académico. En razón a que el proyecto de Especialización se enfoca prioritariamente a la cualificación de la práctica profesional del licenciado en matemáticas, el trabajo anteriormente mencionado se centrará en la construcción de vías didácticas para el tratamiento de nociones específicas de la matemática escolar en algún nivel particular de la educación básica o media.

Control del flujo de estudiantes

El cupo máximo para cada núcleo es de veinticinco estudiantes, seleccionados entre los aspirantes que cumplan los requisitos de admisión establecidos para el proyecto curricular. 
El proyecto de Especialización adopta el sistema de promoción continua. Ningún núcleo es prerrequisito de otro, los estudiantes podrán seleccionarlos e inscribirlos de acuerdo a sus intereses personales.

Para aprobar cualquier núcleo se requiere el cumplimiento de los requisitos específicos correspondientes, situación que se traduce en una nota mínima estipulada en la reglamentación académica de la Universidad. En el evento en que no se hayan cumplido, a satisfacción los requisitos y se hayan agotado todos los medios de recuperación establecidos, se reprobará el núcleo. En tal caso, el estudiante debe cursar y aprobar otro núcleo del plan de estudios.

\section{LA EVALUACIÓN EN EL PROYECTO}

La evaluación es, en este proyecto curricular, un elemento dinamizador fundamentalmente útil, tanto para el estudiante como para el cuerpo profesoral que les permitirá una conceptualización de los procesos vividos por cada uno de ellos en el programa y que alimentará recurrentemente el proceso para la toma de decisiones sobre el proyecto curricular mismo.

El modelo de evaluación que se acoge, en coherencia con el PEI, hace realidad el criterio según el cual la evaluación es un mecanismo de orientación y en concordancia con el sentido pedagógico del proyecto curricular, se acoge el enfoque de evaluación formativa, en el cual la evaluación se realiza para favorecer los procesos de adquisición de competencias en los alumnos.

Se entiende la evaluación, en el proyecto de Especialización, como la reflexión sistemática, integral, dinámica y formativa sobre los procesos desarrollados en los diferentes espacios académicos, que busca apreciar, estimar y valorar en forma comprensiva y participativa los avances conceptuales y metodológicos de los estudiantes en lo referente a la formulación, desarrollo y sustentación de los proyectos de aula que presentan cada trimestre, además del cumplimiento de los requerimientos específicos para cada uno de los núcleos que conforman el plan de estudios del proyecto.

Los instrumentos que se utilicen para la evaluación en los diferentes espacios y momentos del desarrollo del proyecto curricular han de ser sensibles al proceso de cambio que viven sus actores, buscarán la expresión y la participación de los mismos más que la inhibición, en la idea de fomentar la modalidad de evaluación participativa como oportunidad para generar alternativas de construcción y autoevaluación, en el sentido de pensar, analizar y actuar.

La aplicación de la evaluación formativa, se asocia en el proyecto con la evaluación continua a través de procedimientos formales e informales que permitirán la formulación de diagnósticos y el uso de la información que se obtenga permitirá ir ajustando para autorregular el proyecto curricular o apartes del mismo a los grupos y a los desarrollos de competencias propuestos. 


\section{BIBLIOGRAFÍA}

GUTIERREZ, A. (ed) (1991). Área de Conocimiento: Didáctica de la Matemática. Colección Cultura y Aprendizaje. Editorial Síntesis

NCTM (1991). 